\title{
APPLIED PROBABILITY
}

COMPLETE AUTHOR AND SUBJECT

INDEX No. 14

2007-2009

JOURNAL OF APPLIED PROBABILITY VOLUMES 44-46

ADVANCES IN APPLIED PROBABILITY VOLUMES 39-41 
Published by the APPLIED PROBABILITY TRUST

School of Mathematics and Statistics

University of Sheffield Sheffield S3 7RH, UK

Copyright (C) 2010 Applied Probability Trust

Printed in Oxfordshire at Information Press 